OPEN ACCESS

Edited by:

Peican Zhu,

Northwestern Polytechnical

University, China

Reviewed by:

Jingrui Sun,

Southern University of Science and

Technology, China

Fujun Lai,

Yunnan University of Finance And

Economics, China

*Correspondence:

Hanchao Yang

yanghanchao44@gmail.com

Specialty section:

This article was submitted to

Social Physics,

a section of the journal

Frontiers in Physics

Received: 05 June 2021

Accepted: 21 July 2021

Published: 12 August 2021

Citation:

Yan X, Yang $H, Y u Z$ and Zhang S (2021) A Network View of Portfolio Optimization Using

Fundamental Information.

Front. Phys. 9:721007.

doi: 10.3389/fphy.2021.721007

\section{A Network View of Portfolio Optimization Using Fundamental Information}

\author{
Xiangzhen Yan ${ }^{1}$, Hanchao Yang ${ }^{1 *}$, Zhongyuan $Y_{u^{2}}{ }^{2}$ and Shuguang Zhang ${ }^{1}$ \\ ${ }^{1}$ Department of Statistics and Finance, School of Management, University of Science and Technology of China, Hefei, China, \\ ${ }^{2}$ School of Systems and Enterprises, Stevens Institute of Technology, Hoboken, NJ, United States
}

This article proposes the use of a novel approach to portfolio optimization, referred to as "Fundamental Networks" (FN). FN is an effective and robust network-based fundamentalincorporated method, and can be served as an alternative to classical mean-variance framework models. As a proxy for a portfolio, a fundamental network is defined as a set of "interconnected" stocks, among which linkages are a measure of similarity of fundamental information and are referred to asset allocation directly. Two empirical models are provided in this paper as applications of Fundamental Networks. We find that Fundamental Networks efficient portfolios are in general more mean-variance efficient in out-ofsample performance than Markwotiz's efficient portfolios. Specifically, portfolios set for profitability goals create excess return in a general/upward trending market; portfolios targeted for operating fitness perform better in a downward trending market, and can be considered as a defensive strategy in the event of a crisis.

Keywords: portfolio optimization, fundamental analysis, financial networks, asset allocation, minimum spanning tree

\section{INTRODUCTION}

The problem of portfolio optimization is one of the most important issues in asset management [1]. Modern Portfolio Theory (MPT) has served as the foundation and industry standard for portfolio optimization, being capable of demonstrating the concepts of diversification and riskreturn efficiency based on a mean-variance framework [2,3]. However, within the last few decades, practitioners and academics have become aware of its drawbacks. First, the high sensitivity of the estimated mean-variance efficient portfolio to estimation errors in expected return may lead to non-robust results [4-12]. Second, the mean-variance optimization requires the inversion of a positive-definite co-variance matrix which would lead to large estimation errors and will further offset the benefits of diversification. Hence, the portfolio cannot be constructed for a large number of stocks [13]. Third, the lack of hierarchical structure in a correlation matrix allows weights to vary freely in unintended ways, which is a root cause to the mean-variance efficient portfolio's instability [14]. Moreover, MPT only involves the analysis of prices and is not able to incorporate the fundamental analysis which has been deemed valuerelevant to stock return by not only well-known investors mentioned earlier, but also multiple researchers in the past [15-19].

To our knowledge, substitution of mean-variance framework has not been well discussed in the area of portfolio optimization. Studies introduced fundamental analysis to mean-variance model are still confined by the drawbacks. The application of financial networks started a new branch in optimizing portfolios. However, few of them conveyed fundamental information. Therefore, the 
main purpose of our research is to bridge portfolio optimization to fundamental analysis with network structure.

In this paper, we propose a novel approach for portfolio optimization, referred to as "Fundamental Networks" (FN), an effective and robust network-based fundamental-incorporated method. It can be used as an alternative to the classical Markowitz's model. Compare to it, FN avoids the estimation errors of expected return and standard deviation,and also removes the requirement of a positive definite co-variance matrix. As a proxy for a portfolio, a fundamental network is defined as a set of "interconnected" stocks, among which linkages are a measure of similarity of fundamental information among stocks. The capital allocated to a stock is proportionate to the concept of weighted degree in network theory.

The fundamental networks bridge portfolios to fundamental information with network theory. The process of portfolio optimization is decomposed into steps as asset selection and capital allocation [20]. iterature referred to this paper is classified as fundamental analysis and financial networks. However, few of them addressed the both steps.

Fundamental analysis is the most common way to assess firm value [19]. However, the application of fundamental analysis in portfolio optimization tends to focus on the stock selection rather than developing an effective and innovative weighting schema based on fundamental information [18, 20-24]. Attempts introduced an additional fundamental condition into Markowitz's model for both optimal selection and allocation. Although these models are improved by selecting stocks of good economic condition for the portfolio, major limitations from Markowitz's model have not been addressed [25-27]. Another area of studies which is related to our work is fundamental indexation, which assigns portfolio weights using metrics in financial statements instead of stock price and capitalization [28-30].

Pioneered by the remarkable work of [31]; network-based methods have found their way into finance literature, and Recent studies such as [32-35] explore their usefulness for optimal investment purposes. Indeed, as noted in [36]; many financial optimization problems including Markowitz's, had an underlying network structure. The topology of network encodes the complex dependency structure of financial equities, extracts hierarchical and clustering properties, and reduces data complexity while preserving fundamental characteristics of information [34]. Because the functionality of complex networks relies on their underlying structure and structural stability, most empirical studies chose the minimum spanning tree (MST) as the base structure for their financial networks [37-41].

In network theory, information about the relative importance of nodes in a network can be obtained through centrality measures, which are the most fundamental and frequently used measures to reveal network structure [42-46]. Investment decisions are usually derived from choosing "central" or "noncentral" assets from the network. "Non-central" investment strategy claims that a portfolio containing the outskirts of the network have greater diversification potential, and thus are exposed to less risk [32, 34, 35, 45]. However, these studies only select stocks, the allocation process is still under the
Markowitz's mean-variance framework, therefore its drawbacks inherited from the framework remain unsolved.

Our proposed FN can address both asset selection and capital allocation for portfolio investment. In the selection stage, a portfolio objective is set, such as maximizing changes of return on equity (ROE). In the allocation stage, we construct a fundamental network with proper network structure. To start with, we define each selected stock as a node in the network, and each node is described using its fundamental information set which may include changes in net profit, asset turnover and leverage ratio. Next, we define potential links in the network using similarity measure of information sets among stocks, similarity measure can be Euclidean distance or other common distance measures. Finally, a network structure such as the minimum spanning tree (MST) is applied to form connections among stocks, and weighted degrees are derived for capital allocation. Network efficiency is considered to be a measure of network instability and error tolerance [47].

Two empirical models are provided in this paper as applications of the FN. Model 1 is based on DuPont framework where the objective of selecting stocks with high $\mathrm{ROE}$, changes in net profit, asset turnover and leverage ratio serve as fundamental information set to describe a node. Model 2 is focused on operating income, where stock nodes are presented as changes of gross profit. Both of these models use Euclidean distance to construct a MST to be used as the desired network structure. We evaluate model performance using Sharpe Ratio from year 2006 to 2018. Model 1 outperforms the mean-variance efficient portfolios for all risk cases, and Model 2 outperforms the mean-variance efficient portfolios in the low-risk cases. In other words, FN efficient portfolios are more mean-variance efficient in out-of-sample performance than Markwotiz's efficient portfolios. In addition, the annual return of Model 1 is positively correlated to the annual return of the S\&P 500 Index, while Model 2 is negatively correlated to that of the S\&P 500 Index. Unlike the one fixed optimization goal of mean-variance framework, FN provide solutions to complex market conditions. In our study, Model 1 is suggested for general market condition, while Model 2 serves as a defensive strategy in the event of a crisis.

The major contributions of our proposed $\mathrm{FN}$ are:

- Proposing a Novel Framework for Portfolio Optimization: creating an network-based fundamentalincorporated method for portfolio optimization, completely different from the classical Markowitz's model. Empirically, the out-of-sample performance is suggested that network efficient portfolios reached higher mean-variance efficiency than Markowitz's solutions.

- Expanding Source of Information: incorporating fundamental information and providing flexibility in selecting portfolio objective. It can be any fundamental variable or financial ratio representing profitability, liquidity, solvency, operating efficiency etc.

- Lifting Restriction: removing portfolio size constraint in mean-variance framework, referred to the positive-definite requirement of co-variance matrix, so that enable largescale portfolio optimization. 


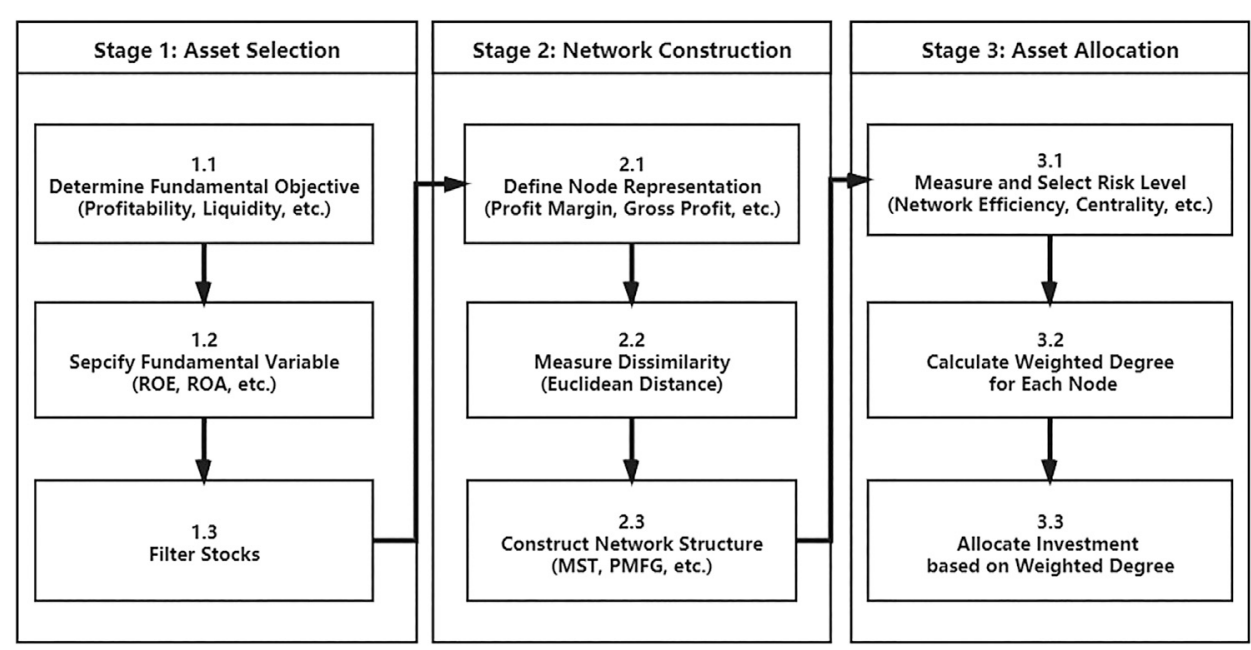

FIGURE 1 | Flowchart for Portfolio Optimization under Fundamental Network Framework. Two empirical examples are specified in Table 2

- Enhancing Robustness of Asset Allocation: increasing stability and robustness of optimal capital allocation on stocks by adopting optimal network structure.

The remainder of the paper is organized as follows: section 2 introduces the general mathematical model of fundamental networks. In section 3, two empirical studies are provided and evaluated. Section 4 presents our conclusions and avenues of future research.

\section{METHODOLOGY}

\subsection{Stock Portfolio as a Network}

In our FN model, the asset selection rule is determined by the chosen fundamental objective. The asset allocation process is to minimize stock similarity under an optimal network structure. For example, ROE is an indicator of corporate profitability. To maximize profitability of a portfolio, we choose the changes of ROE as the fundamental objective and filter a set of high performance companies out of the entire market. We then construct a network based on fundamental information set, using the same example as in Model 1. Elements in the set include changes in net profit, asset turnover and leverage ratio among others. The allocation result is expected to be positively correlated to the weighted degree of each node in the network.

The main novelty of this paper lies in the flexibility choosing objective, information sets and risk measures based on fundamentals. Objectives and information sets can be any fundamental variable or financial ratio representing profitability, liquidity, solvency, operating efficiency etc. Common network structures can refer to, but are not limited to minimum spanning trees, planar maximally filtered graphs, market graphs [48]. Variations in node representation, network structure and distance definition allow a variety of potential risk measures to be applied. Such risk measures can be derived as centrality, assortativity, and network efficiency among others.
Since, centrality, assortativity and network efficiency are measures of similarity and stability in graph theory, these can be considered as diversification and robustness of allocation in portfolio theory $[33,34]$.

\subsection{Fundamental Network Framework}

In this paper, we introduce the concept of "Fundamental Network Framework" as a general process equating portfolio optimization with finding optimal network topology features (Figure 1). The framework is consisted of three stages: asset selection, fundamental network construction and asset allocation.

\subsection{Fundamental Network Construction}

According to Figure 1, Stage 1 and 2 is to define and construct fundamental networks by modeling a portfolio with topological features. A fundamental network is referred to a connected, undirected and weighted graph $G(V, E, W)$.

Definition 1 (Fundamental Network) Define a connected, undirected and weighted graph

$$
G(V, E, W)
$$

where $V=\left\{v_{1}, \ldots, v_{n}\right\}$ denotes a set of stocks. Every stock and its fundamental/financial ratio information is represented as a node. $E=\left\{e_{1}, \ldots, e_{n}\right\}$ is the edge set. $W$ is the weighted adjacency matrix and $w_{i, j} \in W, w_{i, j}$ represents the edge weight between nodes $v_{i}$ and $v_{j}$.

In stage 1 (Figure 1), we need to define the fundamental objective such as profitability, and then refer a specific variable for the objective. In this paper, we only allow one dimension objective. Definition 2 is to mapping a stock to the fundamental variable. For instance, ROE is selected to describe profitability noted as the utility $U=u(V)=R O E$.

Definition 2 (Utility) Define a utility as

$$
U=u(V)
$$

where $u()$ is a utility function. 


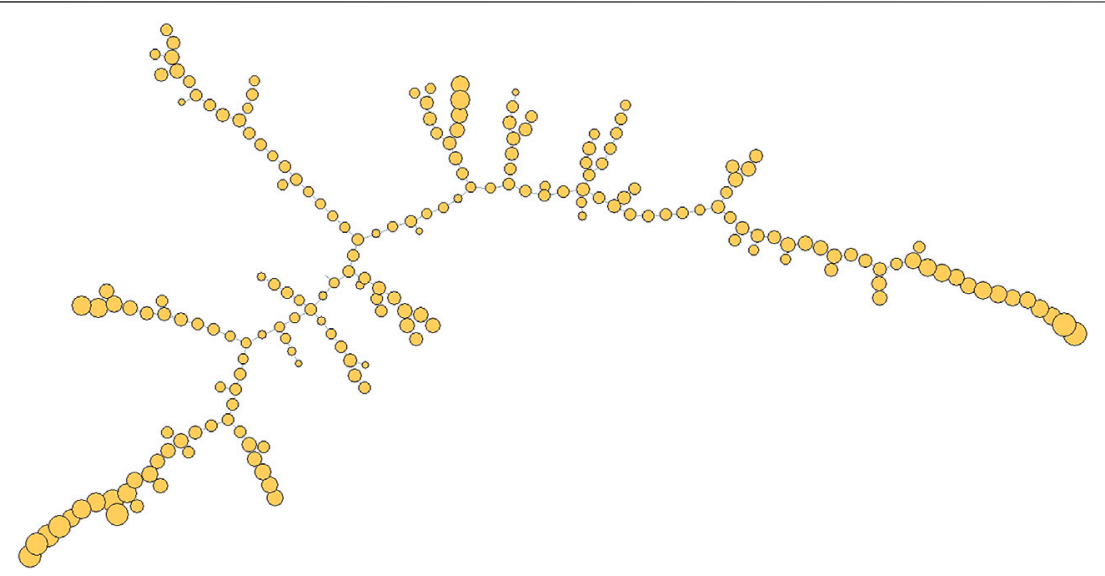

FIGURE 2 | Fundamental Network: Model 1, 200 stocks in 2009. Size of node is positively correlated to weighted degree and investment allocation.

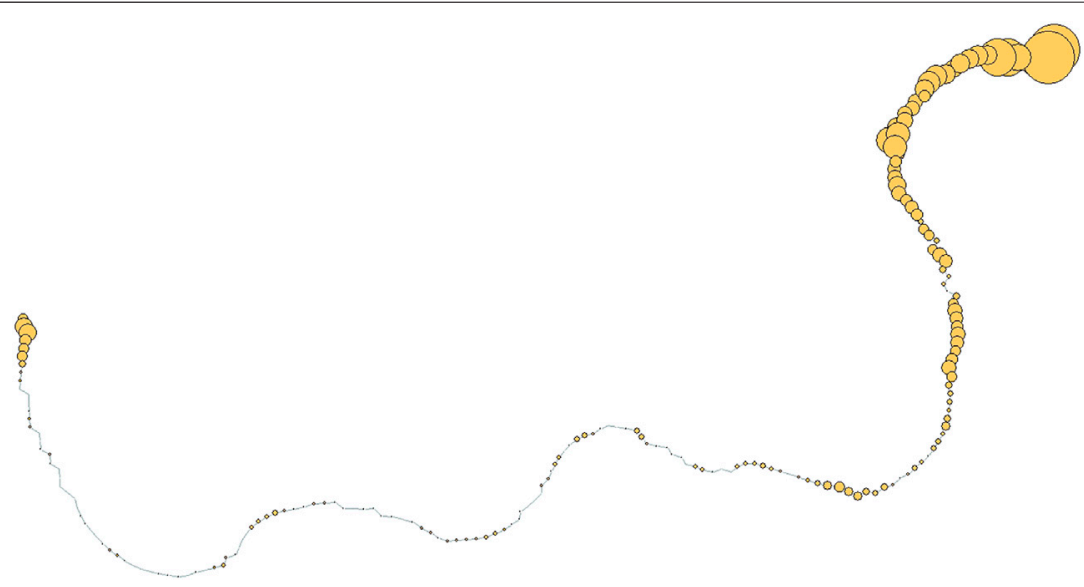

FIGURE 3|Fundamental Network: Model 2, 200 stocks in 2009. Weighted degree is higher for the nodes (larger) near the end points than those in the middle since no hierarchical structure is detected.

Then filter stocks with the variable selected to establish a stock pool for later optimization. Notice that $V_{U}$ are the selected stocks based on $U$ and $U_{\text {threshold }}$. It can represent any predetermined objective such as profitability, liquidity, solvency, or operating efficiency.

Definition 3 (Stock Pool) Select a subset of stocks satisfying

$$
V_{U} \in V, \forall v \in V_{U}, u(v) \geq U_{\text {threshold }}
$$

where $U_{\text {threshold }}$ is a threshold level of $U$.

Stage 2 is mainly to construct fundamental networks based on selected stock pool. Specifically, each node represents the fundamental(s) of a stock. A linkage between two nodes represents the dissimilarity of the corresponding stocks. In this paper, dissimilarity measure is referred to Euclidean distance.

Definition 4 (Fundamental Node) Define $V=\left\{v_{1}, \ldots, v_{n}\right\}, a$ node is

$$
v_{i}=\left\{I_{(i, q)} \mid q=1,2, \ldots, m\right\}
$$

where $v_{i}$ is the fundamental information set of stock $k_{i}$ and $I_{(i, q)}$ is referred to qth fundamental variable representing a stock. $I_{(i, q)}$ ranges from financial ratios to the variables in the financial statements such as leverage ratio, net income, $\Delta$ grossprofit, etc. Note that $m$ is noted as the number of variables to define a node.

Definition 5 (Dissimilarity) Define Euclidean distance as the measure of dissimilarity between two nodes $i, j, D=\{d(i, j), \forall i, j \in$ $[1, n]\}$.

$$
d(i, j)=\sqrt{I_{(i, q)}-I_{(j, q)}}, q=1,2, \ldots, m ;
$$

A network with the set $V_{U}$ can be constructed by Definition 1-3. Given node set $V_{U}$, network structure $E$ is derived from network generation algorithms such as small-world networks, scale-free networks and minimum spanning tree etc. The 
TABLE 1 | Performance Comparison.

\begin{tabular}{|c|c|c|c|c|c|c|c|c|c|}
\hline & $\hat{\mathbf{R}}$ & & & $\hat{\sigma}$ & & & Sharpe $_{t}$ & & \\
\hline Year & Model 1 & Model 2 & MV & Model 1 & Model 2 & MV & Model 1 & Model 2 & MV \\
\hline 2007 & -0.10 & 0.09 & -0.10 & 0.25 & 0.22 & 0.22 & -0.40 & 0.40 & -0.48 \\
\hline 2008 & -0.81 & -0.28 & -0.41 & 0.55 & 0.37 & 0.43 & -1.48 & -0.75 & 0.94 \\
\hline 2009 & 0.94 & 0.34 & 0.41 & 0.43 & 0.23 & 0.18 & 2.20 & 1.45 & 2.24 \\
\hline 2010 & 0.13 & 0.28 & 0.26 & 0.24 & 0.22 & 0.31 & 0.55 & 1.30 & 0.82 \\
\hline 2011 & 0.21 & 0.06 & 0.16 & 0.38 & 0.23 & 0.28 & 0.55 & 0.27 & 0.58 \\
\hline 2012 & 0.30 & 0.10 & 0.15 & 0.22 & 0.15 & 0.23 & 1.34 & 0.65 & 0.68 \\
\hline 2013 & 0.54 & 0.20 & 0.14 & 0.19 & 0.13 & 0.15 & 2.90 & 1.58 & 0.93 \\
\hline 2014 & 0.22 & 0.07 & 0.07 & 0.17 & 0.12 & 0.23 & 1.31 & 0.59 & 0.31 \\
\hline 2015 & -0.01 & 0.09 & -0.08 & 0.21 & 0.21 & 0.23 & -0.05 & 0.43 & -0.33 \\
\hline 2016 & 1.12 & 0.00 & 0.14 & 0.43 & 0.15 & 0.16 & 2.60 & -0.02 & 0.88 \\
\hline 2017 & 0.27 & 0.17 & 0.09 & 0.15 & 0.17 & 0.20 & 1.83 & 0.99 & 0.44 \\
\hline 2018 & 0.05 & 0.02 & -0.41 & 0.21 & 0.24 & 0.32 & 0.25 & 0.09 & -1.26 \\
\hline Average & 0.24 & 0.10 & 0.04 & 0.29 & 0.20 & 0.25 & 0.97 & 0.58 & 0.32 \\
\hline
\end{tabular}

On average, both Model 1 and 2 are more mean-variance efficient than the benchmark. Model 1 is featured with high return and Model 2 has the lowest volatility.

TABLE 2 | Model Specifications.

\begin{tabular}{|c|c|c|c|}
\hline FN framework & Steps & Model 1 & Model 2 \\
\hline \multirow[t]{2}{*}{ Stage 1} & Fundamental Objective & Profitability & Operating Fitness \\
\hline & Fundamental Variable & $\Delta \mathrm{ROE}$ & $\Delta$ Operating Income \\
\hline \multirow[t]{5}{*}{ Stage 2} & Node Representation & $\Delta$ Profit Margin & $\Delta$ Gross Profit \\
\hline & & $\Delta$ Asset Turnover & \\
\hline & & $\Delta$ Leverage Ratio & \\
\hline & Dissimilarity Measure & Euclidean & Euclidean \\
\hline & Network Structure & MST & MST \\
\hline \multirow[t]{2}{*}{ Stage 3} & Risk Measure & TNE & TNE \\
\hline & Allocation & Weighted Degree & Weighted Degree \\
\hline
\end{tabular}

In accordance with Figure 1, this table specifies fundamental information and network related variables for different optimal objectives. $\Delta$ is the difference of the variable between yeart and year $\mathrm{t}-1$. The FN framework allows nodes to be defined in multi-dimensional space such that Model 1 is in I $\mathrm{R}^{3}$ and Model 2 is in I R.

topology features of $G\left(V_{U}, E, W\right)$ vary when choosing different network models. It is found a more complex topology often comes with a greater systemic risk $[37,49,50]$ and a structural optimized network structure will also lead to an optimized portfolio [35, 51].

Fundamental network structural stability and dissimilarity in graph theory is defined in our model as portfolio risk in portfolio theory. Network efficiency is considered to be a risk tolerance measure in graph theory [47]. By bridging stock portfolios to networks, we are able to use network efficiency as a risk measure of a portfolio.

In this paper, we use minimum spanning tree (MST) as the base network structure. A minimum spanning tree (MST) is a tree $T\left(V_{U}, E_{T}, W_{T}\right) \in G(V, E, W)$ with minimum total edge weight.

Definition 6 (Minimum Spanning Tree) Given a connected, undirected weighted graph $G\left(V_{U}, E, W\right)$, the minimum spanning tree (MST) is a tree $T\left(V_{U}, E_{M S T}, W_{M S T}\right) \subseteq G$ with minimum total edge weight defined as:

$$
w_{M S T}=\sum_{e_{i, j} \in E_{M S T}} w_{i, j}, \forall \mathcal{G} \subseteq G
$$

where $\mathcal{G}$ is subgraph of $G$ and $E_{M S T} \subseteq E, W_{M S T} \subseteq W$.
2.4 Risk Measurement and Asset Allocation In stage 3 (Figure 1), after the network is constructed, we introduce total network efficiency (TNE), which is a measure of systemic attack and error tolerance, as the risk measure in our model. A network with higher network efficiency has a higher tolerance to errors and attacks [47].

Definition 7 Total Network Efficiency (TNE)

$$
\operatorname{TNE}(G)=\sum_{i \neq j \in G} \frac{1}{d_{i, j}}
$$

where $d_{i, j}$ is the distance of all paths from node $v_{i}$ to $v_{j} \in G\left(V_{U}, E\right.$, $W), \forall i \neq j$. Note that $G\left(V_{U}, E, W\right)$ is a undirected graph, so $d_{i, j}=d_{j, i}$.

TNE is positively related to the number of nodes and edges since it is defined as the sum of reciprocal of all edges in a network. Therefore, increasing size of an FN will increase its TNE, indicating a reduction of instability and an increase in diversification.

In addition, given a certain number of nodes, a minimum spanning tree has the maximum of TNE $\forall \mathcal{G} \in G$. Mathematically, according to Definition 7, a minimum spanning tree $T$ minimizes $\sum_{i, j} d_{i, j}, \forall i, j \in G$ such that $T N E_{T} \geq T N E_{\mathcal{G}}, \forall \mathcal{G} \in G$, where $\mathcal{G}$ is arbitrary subgraph of $G$. Given a total number of $n$ stocks where 


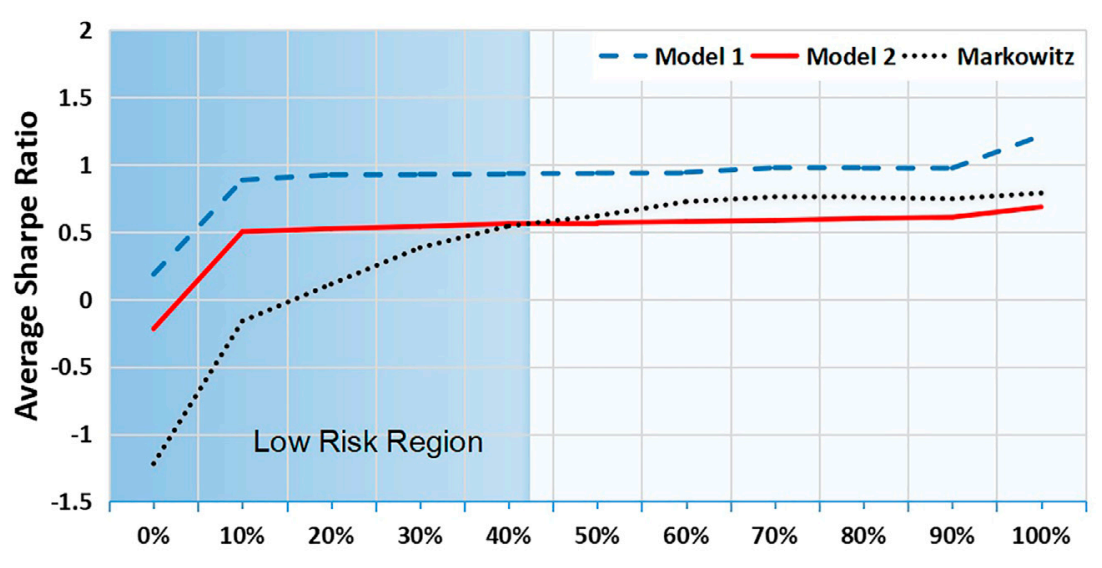

q-th Percentile Normalized Risk

FIGURE 4 | Fundamental Network Efficient Frontiers Model Out-of-Sample Performance. Model 1 (blue) is generally more mean-variance efficient than the benchmark while Model 2 (red) only outperforms in the low risk region ( $q \leq 45 \%$ ). Mean-variance efficient frontier is the benchmark (black).

TABLE 3 | Regressions Analysis and Diagnostics.

\begin{tabular}{ccccc} 
& $\boldsymbol{\beta}$ Coefficient & $\boldsymbol{\alpha}$ & $\boldsymbol{R}^{\mathbf{2}}$ & $\boldsymbol{p}$-value \\
\hline Model 1 & 0.7812 & 0.0622 & 0.2205 & $3.172 \mathrm{E}-15$ \\
Model 2 & -0.5435 & 0.044 & 0.1102 & $6.73 \mathrm{E}-08$
\end{tabular}

A positive $\beta$ indicates that the premium between Model 1 and Markowitz's is positively correlated with market return while Model 2 is opposite with a negative $\beta$.

$V_{U_{i}}=\left\{v_{1}, \ldots, v_{n}\right\}, u\left(v_{i}\right) \geq U_{\text {threshold }}$, the minimum spanning tree set $T=\left\{T_{i}\left(V_{U_{i}}, E_{i}, W\right)\right\}, i=1, \ldots, n$ are the portfolios with maximized.

Finally, investment allocation is calculated from the weighted degree of the nodes in the network. From a network perspective, a stock with high weighted degree is systemically important as it is assigned a large portion of investment.

Definition 8 (Investment Allocation) Given a connected weighted graph.

$G\left(V_{U}, E, W\right), V_{U}=\left\{v_{1}, \ldots, v_{n}\right\}$ is a n-stocks portfolio satisfying $\forall v_{i} \in V_{U}, U\left(v_{i}\right) \geq U_{\text {threshold, }}$ where $v_{i}$ represents the fundamental information set of a stock $I_{i}$.

Define $X=\left(x_{1}, x_{2}, \ldots, x_{n}\right)$ as the vector of investment allocation, $x_{i}$ as the proportion of investment in stock $i$, and $\sum_{i=1}^{n} x_{i}=1$.

$$
x_{i}=\frac{\sum_{j \in N\left(v_{i}\right)} w_{i, j}}{\sum_{i, j \in V} w_{i, j}},
$$

where $N\left(v_{i}\right)$ is the neighbor set of $v_{i}, w_{i, j}$ represents the edge weight between two stocks' fundamentals $v_{i}$ and $v_{j}$.

\section{EMPIRICAL ANALYSIS AND RESULTS}

In this section, we present two empirical solutions in portfolio optimization as applications of Fundamental Networks. While minimizing fundamental network structural instability, the models are distinct from their fundamental objectives and node variables to adapt to different market scenarios. Our optimized portfolios outperforms Markwotiz's under certain market conditions.

\subsection{Data and Models}

Financial statement data are from the Compustat database,the sampled stocks are S\&P 1,500 index members from 2006-2018. There are around 550 to 580 stocks each year after excluding those missing necessary data. The backtest started from 04/15/ 2007 and the positions are adjusted at the first trading day right after each April 15th when all companies had released their financial statements. Risk free rate refers to 1-year t-bill rate. Price returns are calculated annually between each April 15th. Fundamental variables are selected from annual financial statements. A detailed description of fundamental variable settings is shown in Table 2. All fundamental values can be found in the supplementary data set.

To illustrate the flexibility of FN framework in selecting portfolio objectives and fundamental information, we introduce two models in the empirical analysis Table 2. Model 1 maximizes company profitability described by changes of Return on Common Equity (ROE). In accordance with DuPont Analysis, each node is defined by changes in net profit, asset turnover and leverage ratio. Model 2 is focused on operating fitness, fundamental objective is defined as changes of operating income, and stock nodes are presented as changes of gross profit.

In the FN models, Euclidean distance is referred to the measure of diversification. Therefore, instead of restricted by the positive-definite requirement of co-variance matrix in mean-variance framework, portfolio size in FN models can be as large as the stock Universe. In our examples, the portfolio size ranges up to around 550 stocks.

The FN networks displayed in Figures 2, 3 are portfolios selected from efficient frontiers of Model 1 and 2 in 2009, 
each consisted of 200 stocks. Each node represents a stock while linkages measure similarity of their fundamental information. Size of node is positively correlated to weighted degree and investment allocation.

Apparently, the structural complexity of the two networks varies significantly that Model 1 has a hierarchical structure and higher interconnectedness than Model 2. This outcome is related to the complexity-stability debate [52] that whether a positive correlation exists between them. Interconnectedness is a key feature in measuring network complexity. In finance, many studies suggested that interconnectedness conveys higher systemic risk in bank networks [53]. According to [54]; there is no economic theory at hand that can be used to answer the question of the optimal level of interconnectedness for a financial system from a general equilibrium perspective. However, our empirical results suggest that the portfolios with high interconnectedness have higher volatility in out-of-sample performance (Table 1).

\subsection{Empirical Results}

The assessment of the performance is based on out-of-sample statistics in the holding period which is 252 trading days. In stead of individual portfolios, this paper is to compare all the portfolios on the efficient frontiers. Therefore, we investigate the performance from both risk and time perspectives.

Specifically, we note Sharpe Ratio as $\operatorname{Sharpe}(q, t)$, where $t$ is the time and $q$ represents $q$ th percentile of the risk distribution, $q=\{0,5 \%, 10 \%, \ldots, 1\}$. To compare models with different risk measures, we normalize risk into $[0,1]$. Then Sharpe ratio can be represented at intervals of $5 \%$ from 0 to 1 , for a total number of 21 risk levels.

$$
\operatorname{Sharpe}_{q}=\frac{1}{n} \sum_{t=1,2, \ldots, n} \operatorname{Sharpe}(q, t)
$$

where Sharpe $_{q}$ is the average Sharpe ratio of $q$ th percentile portfolios over time $t$.

For example, efficient frontiers are constructed for each year. Sharpe $(5 \%, 2008)$ is referred to Sharpe ratio of the portfolio on the frontier with the lowest $5 \%$ risk at the year 2008. Sharpe $5 \%$ is the average of all the portfolios with lowest $5 \%$ risk from the years between 2007-2019 Figure 4.

In addition, we introduce Sharpe $\hat{e}_{t}$ as the average of Sharpe ratios for all portfolios on the efficient frontier at time t. Similarly, $R$ and $\hat{\sigma}$ represent average return and volatility of the efficient frontier at time $t$.

In Table 1, the Model 1 significantly outperforms Markwotiz's in the year of 2012-2014, 2016, and 2017. These years are often considered to be "good" years where the market earnings growth are positive. The model 2 maximizes operating income and outperforms Markowitz's in "bad" years (2007, 2008, 2015, 2018). The result is consistent with [55] who suggested that high operating performance companies have low volatility and outperforms high volatility stocks.

Define $R=R(q, t), \forall q, t$ to be the actual return of the efficient frontier for all time t; $R_{0}=R_{0}(q, t), \forall q, t$ is the actual return of mean-variance efficient frontier for all time $t ; R_{\text {Market }}$ is the annual return of the stock market which is represented by the annual return of the S\&P 500 Index in this paper.
Let the premium between fundamental network frontier and mean-variance frontier to be:

$$
R(q, t)-R_{0}(q, t)=\beta R_{\text {Market }}(q, t)+\alpha, \forall q, t
$$

As a result, Model 1 creates excess return when market grows but may suffer loss when market drops. Meanwhile, Model 2 has a negative $\beta$ which means it outperforms Markowitz's when market goes down. Model 1 refers to an aggressive strategy by optimizing company profitability. Model 2 is a defensive strategy with emphasis on operating income (Table 3).

\section{CONCLUSION AND DISCUSSION}

We propose a novel approach, named Fundamental Networks (FN), for asset allocation under a network framework, which allows network stability measures to become applicable for portfolio optimization. FN avoids confrontation of estimation errors and the positive definite requirement of the covariance matrix in mean-variance framework. Our FN model provides robust and well-diversified solutions for investment.

Two examples with different fundamental objectives and variables are demonstrated in the paper. We conclude that network efficient portfolios, when properly defined with fundamental variables, are also mean-variance efficient in out-of-sample performance under certain market conditions. Moreover, our approach is adaptive to different market conditions. Portfolios set for profitability goals create excess return in an upward trending market, and outperform Markowitz's benchmark when averaged across years. On the other hand, portfolios targeted for operating fitness produce better returns in the down trending market, and can be used as a defensive strategy in times of crisis. This empirical result also suggests that fundamental networks with lower interconnectedness are less volatile than those with higher complexity.

In conclusion, $\mathrm{FN}$ takes into consideration all filtered stocks and their interconnections, including some stocks which are insignificant but indispensable for the diversification of portfolios. The fundamentals-integrated network reveals both interesting known structures (similarity among stocks) and other structural patterns that are typically lost in the mean-variance framework. When combined with fundamentals and financial ratio information, such patterns can be developed as strategies under different market conditions. This coherent and principled network approach should prove useful for various forms of portfolio construction not limited to fundamental information. More generally, the proposed model is expected to be applied to other complex systems, because of its ability to generalize and connect networks from arbitrary data sets.

There are several avenues for future work. Firstly, a theoretical interpretation of fundamental networks is desired. Secondly, we can investigate, compare, discover patterns for optimal solutions with various fundamental objectives and variables. Thirdly, we can test and analyze more network structures (e.g., planar maximally filtered graph, market graph, maximum cliques among others), and network topological features (e.g., density, assortativity and community structure and others). Finally, fundamental networks open the door for multi-layer stock 
networks modeling fundamental-price dynamics, a potential interpretation for market efficiency.

\section{DATA AVAILABILITY STATEMENT}

The original contributions presented in the study are included in the article/Supplementary Material, further inquiries can be directed to the corresponding author.

\section{REFERENCES}

1. Elton EJ, Gruber MJ, Brown SJ, and Goetzmann WN. Modern Portfolio Theory and Investment Analysis. New York: John Wiley \& Sons (2009).

2. Markowitz H. Portfolio Selection. J Finance (1952) 7:77. doi:10.2307/2975974

3. Markowitz HM. Portfolio Selection: E cient Diversification of Investments. Cowles Foundation Monograph 16 (1959). Diversification, 1959.

4. Mainik G, Mitov G, and Rüschendorf L. Portfolio Optimization for HeavyTailed Assets: Extreme Risk index vs. Markowitz. J Empirical Finance (2015) 32:115-34. doi:10.1016/j.jempfin.2015.03.003

5. Kolm PN, Tütüncü R, and Fabozzi FJ. 60 Years of Portfolio Optimization: Practical Challenges and Current Trends. Eur J Oper Res (2014) 234:356-71. doi:10.1016/j.ejor.2013.10.060

6. Chopra VK, and Ziemba WT. The Effect of Errors in Means, Variances, and Covariances on Optimal Portfolio Choice. In: Handbook of the Fundamentals of Financial Decision Making: Part I (World Scientific) (2013). p. 365-73. (Singapore: World Scientific). doi:10.1142/9789814417358 0021

7. Jagannathan R, and Ma T. Risk Reduction in Large Portfolios: Why Imposing the Wrong Constraints Helps. J Finance (2003) 58:1651-83. doi:10.1111/15406261.00580

8. Jorion P. Bayesian and Capm Estimators of the Means: Implications for Portfolio Selection. J Banking Finance (1991) 15:717-27. doi:10.1016/03784266(91)90094-3

9. Michaud RO. The Markowitz Optimization Enigma: Is 'Optimized' Optimal? Financial Analysts J (1989) 45:31-42. doi:10.2469/faj.v45.n1.31

10. Jorion P. Bayes-stein Estimation for Portfolio Analysis. J Financial Quant Anal (1986) 21:279-92. doi:10.2307/2331042

11. Jorion P. International Portfolio Diversification with Estimation Risk. J Bus (1985) 58:259-78. doi:10.1086/296296

12. Barry CB. Portfolio Analysis under Uncertain Means, Variances, and Covariances. J Finance (1974) 29:515-22. doi:10.1111/j.15406261.1974.tb03064.x

13. López de Prado M. Building Diversified Portfolios that Outperform Out of Sample. Jpm (2016) 42:59-69. doi:10.3905/jpm.2016.42.4.059

14. Raffinot T. Hierarchical Clustering-Based Asset Allocation. Jpm (2017) 44: 89-99. doi:10.3905/jpm.2018.44.2.089

15. Nissim D, and Penman SH. Ratio Analysis and Equity Valuation: From Research to Practice. Rev Account Stud (2001) 6:109-54. doi:10.1023/a: 1011338221623

16. Sloan RG. Do stock Prices Fully Reflect Information in Accruals and Cash Flows about Future Earnings? Account Rev (1996) 289-315.

17. Lev B, and Thiagarajan SR. Fundamental Information Analysis. J Account Res (1993) 31:190-215. doi:10.2307/2491270

18. Ou JA, and Penman SH. Financial Statement Analysis and the Prediction of Stock Returns. J Account Econ (1989) 11:295-329. doi:10.1016/0165-4101(89) 90017-7

19. Ball R, and Brown P. An Empirical Evaluation of Accounting Income Numbers. J Account Res (1968) 6:159-78. doi:10.2307/2490232

20. Edirisinghe NCP, and Zhang X. Generalized Dea Model of Fundamental Analysis and its Application to Portfolio Optimization. J Banking Finance (2007) 31:3311-35. doi:10.1016/j.jbankfin.2007.04.008

21. Ho CTB. Performance Measurement Using Data Envelopment Analysis and Financial Statement Analysis. Ijor (2007) 2:26-38. doi:10.1504/ijor.2007.011441

\section{AUTHOR CONTRIBUTIONS}

$\mathrm{XY}$ and HY contributed to conception and design of the study. XY organized the database. HY performed the statistical analysis. XY, HY, ZY and SZ wrote sections of the manuscript. $\mathrm{XY}, \mathrm{HY}$, and $\mathrm{ZY}$ wrote sections of the manuscript. All authors contributed to manuscript revision, read, and approved the submitted version.

22. Küçükşahin $H$, and Coşkun E. The Performance of Fundamental Indexes: An Application on Istanbul. Ege Acade Rev (2020) 20:1-18. doi:10.21121/ eab.595407

23. Xidonas P, Mavrotas G, and Psarras J. A Multicriteria Methodology for Equity Selection Using Financial Analysis. Comput Operations Res (2009) 36: 3187-203. doi:10.1016/j.cor.2009.02.009

24. Yu Z, Serban N, and Rouse WB. The Demographics of Change: Enterprise Characteristics and Behaviors that Influence Transformation. J Enterprise Transformation (2013) 3:285-306. doi:10.1080/19488289.2013.860346

25. Tarczyński W. Different Variants of Fundamental Portfolio. Folia Oeconomica Stetinensia (2014) 14:47-62.

26. Lyle MR, and Yohn TL. Fundamental Analysis and Mean-Variance Optimal Portfolios. Forthcoming, The Accounting Review (2019). Lakewood Ranch: American Accounting Association.

27. Zhang H, and Yan C. Modelling Fundamental Analysis in Portfolio Selection. Quantitative Finance (2018) 18:1315-26. doi:10.1080/14697688.2017.1418520

28. Blitz D, and Swinkels L. Fundamental Indexation: An Active Value Strategy in Disguise. J Asset Manag (2008) 9:264-9. doi:10.1057/jam.2008.23

29. Arnott RD, Hsu J, and Moore P. Fundamental Indexation. Financial Analysts J (2005) 61:83-99. doi:10.2469/faj.v61.n2.2718

30. Hsu JC. Cap-weighted Portfolios Are Sub-optimal Portfolios. J investment Manage (2004) 4

31. Mantegna RN. Hierarchical Structure in Financial Markets. Eur Phys J B (1999) 11:193-7. doi:10.1007/s100510050929

32. Peralta G, and Zareei A. A Network Approach to Portfolio Selection. J empirical Finance (2016) 38:157-80. doi:10.1016/j.jempfin.2016.06.003

33. Kaya H. Eccentricity in Asset Management. Jntf (2015) 1:1-32. doi:10.21314/ jntf.2015.003

34. Pozzi F, Di Matteo T, and Aste T. Spread of Risk across Financial Markets: Better to Invest in the Peripheries. Sci Rep (2013) 3:1665. doi:10.1038/ srep01665

35. Onnela JP, Chakraborti A, Kaski K, Kertész J, and Kanto A. Dynamics of Market Correlations: Taxonomy and Portfolio Analysis. Phys Rev E Stat Nonlin Soft Matter Phys (2003) 68:056110. doi:10.1103/PhysRevE.68.056110

36. Nagurney A. Networks in Economics and Finance in Networks and beyond: A Half century Retrospective. Networks (2019) 77 (1), 50-65. (New York: Wiley Online Library).

37. Khashanah K, and Yang H. Evolutionary Systemic Risk: Fisher Information Flow Metric in Financial Network Dynamics. Physica A: Stat Mech its Appl (2016) 445:318-27. doi:10.1016/j.physa.2015.10.012

38. Heiberger RH. Stock Network Stability in Times of Crisis. Physica A: Stat Mech its Appl (2014) 393:376-81. doi:10.1016/j.physa.2013.08.053

39. Eom C, Kwon O, Jung W-S, and Kim S. The Effect of a Market Factor on Information Flow between Stocks Using the Minimal Spanning Tree. Physica A: Stat Mech its Appl (2010) 389:1643-52. doi:10.1016/j.physa.2009.12.044

40. Coelho R, Gilmore CG, Lucey B, Richmond P, and Hutzler S. The Evolution of Interdependence in World Equity Markets-Evidence from Minimum Spanning Trees. Physica A: Stat Mech its Appl (2007) 376:455-66. doi:10.1016/j.physa.2006.10.045

41. Situngkir H, and Surya Y. On Stock Market Dynamics Through Ultrametricity Of Minimum Spanning Tree. Tech. Rep. Bandung: Bandung Fe Institute (2005).

42. Zhu S, Kou M, Lai F, Feng Q, and Du G. The Connectedness of the Coronavirus Disease Pandemic in the World: A Study Based on Complex 
Network Analysis. Front Phys (2021) 8:642. doi:10.3389/ fphy.2020.602075

43. Gao C, Su Z, Liu J, and Kurths J. Even central Users Do Not Always Drive Information Diffusion. Commun ACM (2019) 62:61-7. doi:10.1145/3224203

44. Newman MEJ. Mathematics of Networks. New palgrave encyclopedia Econ (2008) 2:1-8. doi:10.1057/978-1-349-95121-5 2565-1

45. Chi KT, Liu J, and Lau FC. A Network Perspective of the Stock Market. J Empirical Finance (2010) 17:659-67.

46. Hu J, Chengyi X, Huijia L, Peican Z, and Wenjun X. Properties and structural analyses of USA's regional electricity market: A visibility graph network approach. Applied Mathematics and Computation (2010) (Elsevier) 385, 125434.

47. Latora V, and Marchiori M. Efficient Behavior of Small-World Networks. Phys Rev Lett (2001) 87:198701. doi:10.1103/physrevlett.87.198701

48. Kalyagin VA, Koldanov AP, Koldanov PA, Pardalos PM, and Zamaraev VA. Measures of Uncertainty in Market Network Analysis. Physica A: Stat Mech its Appl (2014) 413:59-70. doi:10.1016/j.physa.2014.06.054

49. Yalamova R, and McKelvey B. Explaining what Leads up to Stock Market Crashes: A Phase Transition Model and Scalability Dynamics. J Behav Finance (2011) 12:169-82. doi:10.1080/ 15427560.2011.602484

50. He J, and Deem MW. Structure and Response in the World Trade Network. Phys Rev Lett (2010) 105:198701. doi:10.1103/physrevlett.105.198701

51. Shi Y, Zheng Y, Guo K, Jin Z, and Huang Z. The Evolution Characteristics of Systemic Risk in China's Stock Market Based on a Dynamic Complex Network. Entropy (2020) 22:614. doi:10.3390/e22060614

52. McCann KS. The Diversity-Stability Debate. Nature (2000) 405:228-33. doi:10.1038/35012234
53. Battiston S, and Martinez-Jaramillo S. Financial Networks and Stress Testing: Challenges and New Research Avenues for Systemic Risk Analysis and Financial Stability Implications (2018). Amsterdam: Elsevier.

54. Martinez-Jaramillo S, Carmona CU, and Kenett DY. Interconnectedness and Financial Stability. J Risk Manage Financial Institutions (2019) 12 (2), 168-83. Henry Stewart Publications.

55. Dutt T, and Humphery-Jenner M. Stock Return Volatility, Operating Performance and Stock Returns: International Evidence on Drivers of the 'low Volatility' Anomaly. J Banking Finance (2013) 37:999-1017. doi:10.1016/ j.jbankfin.2012.11.001

Conflict of Interest: The authors declare that the research was conducted in the absence of any commercial or financial relationships that could be construed as a potential conflict of interest.

Publisher's Note: All claims expressed in this article are solely those of the authors and do not necessarily represent those of their affiliated organizations, or those of the publisher, the editors and the reviewers. Any product that may be evaluated in this article, or claim that may be made by its manufacturer, is not guaranteed or endorsed by the publisher.

Copyright (c) 2021 Yan, Yang, Yu and Zhang. This is an open-access article distributed under the terms of the Creative Commons Attribution License (CC BY). The use, distribution or reproduction in other forums is permitted, provided the original author(s) and the copyright owner(s) are credited and that the original publication in this journal is cited, in accordance with accepted academic practice. No use, distribution or reproduction is permitted which does not comply with these terms. 